\title{
Influence of Magnetic Field Ripple on the Intrinsic Rotation of Tokamak Plasmas
}

\author{
M. F. F. Nave, ${ }^{1}$ T. Johnson, ${ }^{2}$ L.-G. Eriksson, ${ }^{3}$ K. Crombé, ${ }^{4}$ C. Giroud,${ }^{5}$ M.-L. Mayoral, ${ }^{5}$ J. Ongena, ${ }^{4}$ A. Salmi, ${ }^{6}$ \\ T. Tala, ${ }^{6}$ and M. Tsalas ${ }^{7}$ \\ ${ }^{1}$ Associação EURATOM/IST, Instituto de Plasmas e Fusão Nuclear-Laboratorio Associado, Lisbon, Portugal \\ ${ }^{2}$ Euratom-VR Association, EES, KTH, Stockholm, Sweden \\ ${ }^{3}$ European Commission, Research Directorate General, B-1049 Brussels, Belgium \\ ${ }^{4}$ Association EURATOM-Belgian State, Brussels, Belgium \\ ${ }^{5}$ Euratom/CCFE Fusion Association, Culham Science Centre, Abingdon, United Kingdom \\ ${ }^{6}$ Association Euratom-Tekes, VTT, P.O. Box 1000, FI-02044 VTT, Finland \\ ${ }^{7}$ Association EURATOM-Hellenic Republic, Attica, Greece \\ (Received 5 February 2010; published 3 September 2010)
}

\begin{abstract}
Using the unique capability of JET to monotonically change the amplitude of the magnetic field ripple, without modifying other relevant equilibrium conditions, the effect of the ripple on the angular rotation frequency of the plasma column was investigated under the conditions of no external momentum input. The ripple amplitude was varied from $0.08 \%$ to $1.5 \%$ in Ohmic and ion-cyclotron radio-frequency (ICRF) heated plasmas. In both cases the ripple causes counterrotation, indicating a strong torque due to nonambipolar transport of thermal ions and in the case of ICRF also fast ions.
\end{abstract}

PACS numbers: 52.55.Fa, 52.25.Fi, 52.30.Cv, 52.55.Dy

Plasma rotation is one of the central topics for toroidal plasma confinement and it is considered to play a key role in tokamak plasma performance. Plasma rotation can have a strong impact on plasma stability and confinement of fusion plasmas, through its ability to stabilize MHD modes [1], affect the strength of transport barriers [2], affect turbulence stabilization due to $E \times B$ shear [3], and reduce the profile stiffness in the presence of anomalous transport [4]. In present day experiments, rotation is often driven by the torques of neutral beam injection (NBI), while in an alpha-heated reactor the momentum input is expected to be small [5]. In reactors with high NBI power, the torque is still expected to be small due to the high injection energy needed for the beam to penetrate deep into the plasma, which reduces the torque per MW of injected power. Thus, there has been a growing interest in the intrinsic rotation of the plasma, which is observed to occur in the absence of momentum sources such as NBI. Besides its relevance for fusion reactors, the residual plasma rotation in confinement configurations, without momentum injection, is an interesting, and not yet understood, problem from a basic physics point of view that has attracted great theoretical and experimental interest in recent years. The extrapolation from intrinsic plasma rotation data observed in several machines suggests that a substantial rotation in the cocurrent direction, i.e., in the direction parallel to the plasma current, will occur in ITER [6]. However, an effect that is still to be accounted for when extrapolating intrinsic rotation observations to ITER comes from the toroidal ripple in the magnetic field, which appears in tokamaks due to the finite number of toroidal magnetic field coils. ITER will have 18 toroidal magnetic field coils and, a ripple amplitude $\delta=\delta B / B$ of $0.5 \%$ to $1.2 \%$ at the edge (depending on the configuration of ferritic inserts) [7] where $B$ is the averaged toroidal magnetic field and $\delta B$ is the Fourier amplitude of the toroidal ripple. Since the ripple breaks the toroidal symmetry, the motion of individual particles may lead to nonambipolar transport that can affect the plasma rotation through the neoclassical toroidal viscosity [8-10].

The effect of ripple on plasma rotation during NBI has been reported from JT-60 [11] and JET [12]. Evidence that ripple transport of fast ions induces edge counterrotation in plasmas with no external momentum input was found in JT-60 experiments with perpendicular NBI [11]. Ripple experiments at JET with NBI heating have shown that increasing ripple reduced rotation in plasmas with injected momentum parallel to the plasma current [12]. In some cases, countercurrent rotation was observed at the edge. The effect of the ripple was comparable in magnitude to the drive from tangential beam injection. However, torques from fast-ions' nonambipolar transport were not enough to completely explain the observations [13], leaving open the question of the role of ripple on thermal ions. The effect of ripple on intrinsic rotation has been recently studied at JET, as reported here. In order to separate ripple fast-ion effects from thermal ion effects and to test existing ripple transport models, plasma rotation was measured in Ohmic and in ion-cyclotron radio-frequency (ICRF) heated plasmas with ITER-relevant ripple levels.

JET has 32 toroidal field coils and, therefore, a very low ripple level. However, due to a unique design feature, ripple can be increased by reducing the current carried in every second coil (Fig. 1). The ripple is largest in the outboard of the plasma where the plasma is close to the toroidal magnetic field coils. At JET the ripple amplitude differs by 2 orders of magnitude between the plasma core and edge. Furthermore, the distance between toroidal field coils is larger on the outboard, than the inboard side. The 


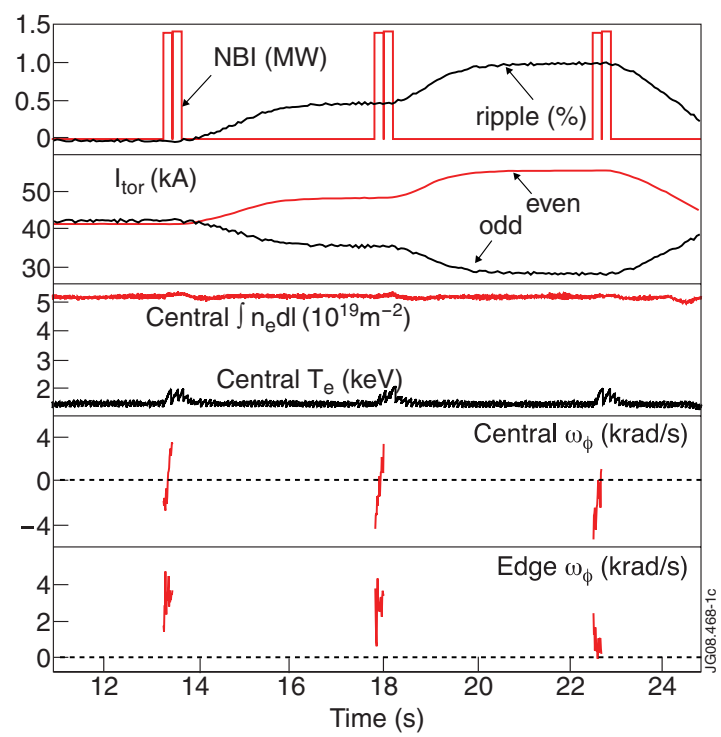

FIG. 1 (color online). Ohmic pulse (\#74758) with three ripple levels. The boxes from top to bottom show: the ripple level and, short NBI pulses for diagnostic purposes; currents at odd and even toroidal coils to provide an average toroidal field at the center of $\left\langle B_{T}\right\rangle=2.2 \mathrm{~T}$ (with $I_{p}=2.1 \mathrm{MA}$ ); central temperature and central line integral density; and central $(R=3.1 \mathrm{~m})$ and edge $(R=3.8 \mathrm{~m}) \mathrm{C}$ toroidal rotation angular frequencies measured by CXRS.

highest ripple inside the plasma is therefore often found at the separatrix near the outboard equatorial plane. Values quoted in this Letter are taken at $R=3.80 \mathrm{~m}, Z=0 \mathrm{~m}$, which is close to the maximum value. In standard operation with 32 coils the ripple amplitude is $\delta=0.08 \%$. This was increased to $\delta=1.5 \%$.

The intrinsic rotation ripple experiments were performed in deuterium for two plasma current values: $I_{p}=$ 1.5 and 2.1 MA, with an average toroidal field at the center of $\left\langle B_{T}\right\rangle=2.2 \mathrm{~T}$, with unbalanced odd and even currents as shown in Fig. 1. The short NBI pulses of 1.4 MW are for diagnostic purposes. The toroidal angular frequency rotation profiles were measured from charge exchange recombination spectroscopy (CXRS) of $\mathrm{C}^{+6}$ [14] during these short NBI pulses, with an integration time of $10 \mathrm{~ms}$. Since NBI at JET provides a cocurrent toroidal momentum source, only measurements taken within the first $20 \mathrm{~ms}$ were used, when NBI momentum was still negligible. In the convention used here, positive rotation means rotation in the cocurrent direction. The difference in toroidal rotation speeds of the D main ion and the C impurity [15] are estimated to be smaller than the CXRS measurement uncertainty [16], which is showed in the figures as shaded areas. The charge exchange measurements in the plasma core were found to be consistent with the observed frequency and direction of propagation of MHD modes, that is the main ion frequency Doppler shifted by the frequency of the MHD instability.

With its usual ripple value, i.e., $\delta=0.08 \%$, JET plasmas with low momentum input, namely, with ICRF [17-
19], lower hybrid [19] and Ohmic heating [20] are observed to rotate with small toroidal angular frequencies, typically $\omega_{\phi} \leq 10 \mathrm{krad} / \mathrm{s}$. For this low ripple level the edge is corotating independent of heating scenario. However, the core of JET plasmas without NBI is either co- or countercurrent rotating. The core of Ohmic plasmas is counterrotating [20], while the core of ICRF heated plasmas is either counter- or corotating depending on plasma current [19] and heating details [18].

The ripple effect on the rotation of Ohmic plasmas is illustrated in Figs. 1 and 2. The temporal evolution of the central $(R=3.1 \mathrm{~m})$ and edge $(R=3.8 \mathrm{~m})$ toroidal rotation frequencies are shown in Fig. 1. The initial central value that was negative, that is countercurrent rotating, became more negative as ripple increased, while the edge corotation decreased and for ripple values $\geq 1 \%$ edge countercurrent rotation is observed. Charge exchange toroidal rotation profiles indicated that the edge and core effects are of the same order. The core effect is also seen in the frequency and direction of propagation of sawtooth pre- and postcursor oscillations observed in the electron temperature signals around the sawtooth inversion radius [Fig. 2(b)]. [Poloidal counterrotation in Ohmic plasmas has also been observed to increase with ripple. However, since the time resolution $(50-100 \mathrm{~ms})$ is too slow, it is not possible to separate ripple effects on NBI fast ions from those on thermal ions.]

The ICRF experiments were performed with hydrogen minority heating in deuterium plasmas using a dipole

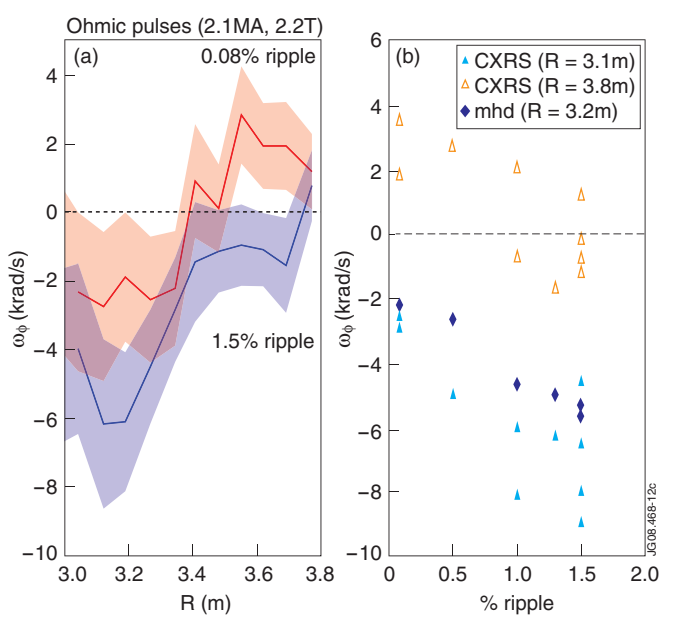

FIG. 2 (color online). (a) $\mathrm{C}$ toroidal rotation angular frequency profiles for Ohmic pulses \#74758 with $\delta=0.08 \%$ and \#74599 with $\delta=1.5 \%, I_{p}=2.1 \mathrm{MA}$ and $\left\langle B_{T}\right\rangle=2.2 \mathrm{~T}$. Here and in the following figures, the profiles shown are the average over the first $20 \mathrm{~ms}$, shaded areas show the measurement uncertainty. (b) $\mathrm{C}$ toroidal rotation angular frequencies as a function of ripple for Ohmic pulses with $2.1 \mathrm{MA}$, at the center $(R=3.1 \mathrm{~m}$, solid triangles) and the edge $(R=3.8 \mathrm{~m}$, open triangles $)$ and, the frequency of sawtooth postcursors (solid kites) that are observed in the electron temperature profiles around the sawtooth inversion radius $R_{\text {inv }}=3.2 \mathrm{~m}$. The sawtooth inversion radius and period remained unchanged with ripple. 
antenna phasing. Since the toroidal field ripple can induce high heat fluxes of fast ICRF accelerated ions to plasma facing components [21] the operation was restricted to modest ICRF power, less than $4 \mathrm{MW}$, and to cyclotron resonances near the plasma core, or on the high-field side of the plasma. Figures 3(a) and 3(b) illustrate the observed effect of toroidal field ripple on ICRF heated plasmas with the ICRF resonance position slightly off axis on the highfield side (position No. 2 in Fig. 4 inset). As the TF ripple increases, both the edge and the core of the plasma counterrotate. Unlike, the Ohmic plasmas where the effect is spatially uniform, the effect is larger in the plasma core. Experimentally, countercurrent rotation was found to depend on ripple, fast-ion losses associated to MHD modes, as well as on local plasma conditions and heating details. For the lower plasma current, $I_{p}=1.5 \mathrm{MA}, H$-mode plasmas were obtained. Core counterrotation was observed to be larger in phases with type III-ELMS as shown in Fig. 3(b). It is not known how the ELM frequency in ELMy $H$ modes affects the intrinsic rotation (as this would require edge CXRS measurements with a higher temporal resolution than available). Thus it is not clear if the difference in rotation between type I and type III-ELM regimes is a pedestal or a density effect. This counterrotation in the core of plasmas with $I_{p}=1.5 \mathrm{MA}$ in type III-ELMs is a factor of 2 larger when compared with $L$-mode plasmas with $I_{p}=2.1$ MA. It seems to confirm the result previously obtained with zero ripple where corotation increased with
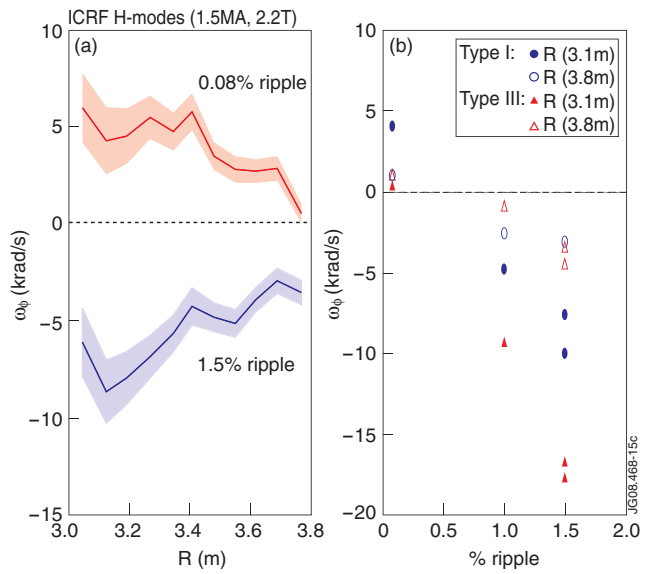

FIG. 3 (color online). (a) $\mathrm{C}$ toroidal rotation angular frequency profiles for ICRF heated $H$-mode plasmas with type I ELMs, $I_{p}=1.5 \mathrm{MA},\left\langle B_{T}\right\rangle=2.2 \mathrm{~T}, P_{\mathrm{ICRF}} \sim 3 \mathrm{MW}$, for two ripple levels. Top: pulse \#74688 with $\delta=0.08 \%$ and $P_{\mathrm{ICRF}}=$ $3.1 \mathrm{MW}$; bottom: pulse $\# 74686 \delta=1.5 \%$ and $P_{\mathrm{ICRF}}=$ 2.9 MW. The plasma center is at $R_{0}=3.02 \mathrm{~m}$, the ICRF resonance is on the high-field side at $R_{\text {res }}=2.71 \mathrm{~m}$ (corresponding to position No. 2 in Fig. 4 inset). (b) $\mathrm{C}$ toroidal rotation angular frequency as a function of ripple for ICRF heated $H$-mode pulses. All pulses have the same resonance position, on the high-field side $\left(R_{\text {res }}=2.71 \mathrm{~m}\right.$, corresponding to position No. 2 in Fig. 4 inset), for the center $(R=3.1 \mathrm{~m})$ and the edge $(R=$ $3.8 \mathrm{~m}$ ), for type I ELM (circles) and type III-ELM (triangles) phases. plasma current [19]. The effect of changing the position of the ICRF resonance was studied in $L$-mode plasmas with $I_{p}=1.5 \mathrm{MA}$. Figure 4 shows the rotation frequency profiles for pulses with $1.5 \%$ ripple. The largest edge and core counterrotation was observed when the ICRF resonance position was on the low-field side, i.e., where the ripple amplitude is stronger. This correlation between the ripple experienced by the fast ions and the rotation indicate that the fast ions receive a torque from the toroidal field coils.

The theory of plasma rotation with toroidal field ripple suggests that, by breaking the toroidal symmetry, the ripple induces radial particle diffusion, which in general is different for ions and electrons [22-24]. This nonambipolar transport gives rise to a charge separation that relaxes the radial electric field and the toroidal rotation toward a steady state where the particle flux is ambipolar. The steady state toroidal plasma rotation was calculated in Refs. [8-10] to be in the counter-current direction. The observations of counter-current rotation reported here are therefore consistent with this predicted direction.

Similar observations have been made on Tore Supra [25]. However, the ripple amplitude in Tore Supra is high enough for significant magnetic wells to form between the toroidal field coils. The transport in the Tore Supra experiments is therefore dominated by particles trapped in these wells $[22,24]$. In machines such as JET or ITER, the ripple is lower and the wells are negligible. Instead the transport is driven by resonant interaction between the ripple and particles following banana orbits [8-10,24]. Thus, the results observed here confirm the theoretical predictions under conditions relevant for ITER.

A neoclassical toroidal viscosity, similar to the one present in ripple experiments, can also be achieved by external application of low- $n$ nonresonant magnetic per-

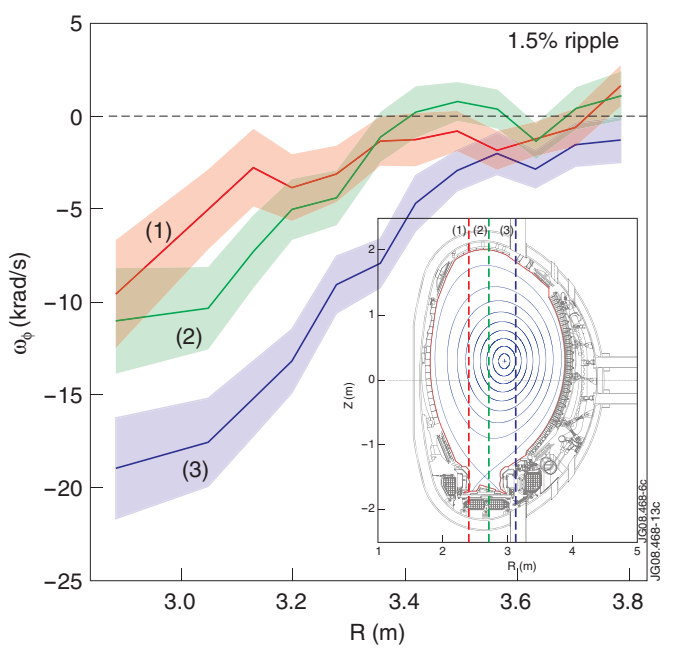

FIG. 4 (color online). $\mathrm{C}$ toroidal rotation angular frequency profiles for $L$-mode pulses with $\delta=1.5 \%, 1.5 \mathrm{MA}, P_{\mathrm{ICRF}} \sim$ $2 \mathrm{MW}$, for different resonance positions: (1) \#77010 with $R_{\text {res }}=$ $2.38 \mathrm{~m}$, (2) \#77014 with $R_{\text {res }}=2.71 \mathrm{~m}$, (3) \#77009 with $R_{\text {res }}=$ $3.13 \mathrm{~m}$. The resonance positions with respect to the magnetic axis $R_{0} \sim 2.92-2.96 \mathrm{~m}$ are shown in the inset. 
turbations, where $n$ is the toroidal mode number. In experiments with externally induced $n=3$ and $n=1$ perturbations, performed at DIII-D [26] and NSTX [27], countercurrent rotation was observed due to the neoclassical toroidal viscosity. In DIII-D, the rotation was found to increase up to a value consistent with the neoclassical residual rotation as predicted in $[8,9,28]$. Thus, countercurrent rotation has been observed under a variety of conditions with nonaxisymmetric perturbations.

While the observations in JET were able to confirm the theoretically predicted direction of rotation, a comparison with the predicted rotation speed is not straightforward. The collisionality $\nu$ of the Ohmic heated pulses is at the lower limit of validity for the $1 / \nu$ transport regime [9]. According to the theory developed in this regime, the residual neoclassical carbon rotation near the edge (normalized radius $\rho=0.9$, or $R \sim 3.8 \mathrm{~m}$ ) of the 2.1 MA pulses shown in Fig. 2(b) is predicted to be $\omega^{* \mathrm{NC}}{ }_{1 / \nu}=3.36 /$ $\left(B_{\theta}\right) \partial_{r} T_{i} \sim-4.5 \pm 2.6 \mathrm{krad} / \mathrm{s}$, where the temperature is taken from CXRS measurements. This is about a factor 2 greater than the fastest rotation measured in Ohmic plasmas at $R=3.8 \mathrm{~m}$. Thus, in Fig. 2(b), when the ripple amplitude is increased the rotation tends to slowly approach the residual rotation $\omega^{* \mathrm{NC}}{ }_{1 / \nu}$. This is consistent with the neoclassical theory in which the intrinsic rotation, the one present in the absence of ripple, is relaxed towards the residual rotation at a rate that is proportional to $\delta^{2}[8,9]$.

In the JET ICRF heated plasmas with enhanced ripple, the toroidal rotation change in the plasma core is larger than in the Ohmic plasmas indicating that the torque source in this case would be less edge localized and that fast-ion as well as thermal ion effects may be involved. In ICRF heated plasmas with ripple where core toroidal rotation becomes more and more negative an inward momentum pinch [29] could also be important. However, an inward pinch cannot explain the reversal of the rotation observed at midradius shown in Fig. 2(a), also observed in ICRF heated plasmas at low plasma currents [19].

In conclusion, ripple has a significant effect on the plasma rotation in the absence of external momentum sources. In both Ohmic and ICRF heated plasmas ripple causes counterrotation. At ITER-relevant ripple values of $\delta=0.5 \%$, JET plasmas with ICRF heating are hardly rotating, while at $\delta=1 \%$, both $L$ and $H$ modes are observed to counterrotate. These results do not fit the intermachine intrinsic rotation scaling of [6]. JET results suggest that ripple can affect rotation in ITER and, it should be taken into account in extrapolation from present data.

This work, supported by the European Communities through ASSOCIATION EURATOM/IST, was carried out within the frameworks of the Instituto de Plasmas e Fusão Nuclear-Laboratório Associado and the European Fusion Development Agreement. It received financial support from Fundação para a Ciencia e Tecnologia (FCT), Portugal. The views and opinions expressed herein do not necessarily reflect those of the European Commission. We thank Professor R. Galvão for his comments. This work was done under the JET-EFDA workprogramme [30].

[1] P. C. de Vries et al., Plasma Phys. Controlled Fusion 38, 467 (1996).

[2] Y. Sakamoto et al., Nucl. Fusion 41, 865 (2001).

[3] T. S. Hahmand and K. H. Burrell, Phys. Plasmas 2, 1648 (1995).

[4] P. Mantica et al., Phys. Rev. Lett. 102, 175002 (2009).

[5] M. N. Rosenbluth and F. L. Hinton, Nucl. Fusion 36, 55 (1996).

[6] J. E. Rice et al., Nucl. Fusion 47, 1618 (2007).

[7] G. Saibene, in Proceedings of the 34th EPS Conference on Plasma Physics, Warsaw, 2007 (ECA, 2007, Warsaw) (European Physical Society, Warsaw, 2007), Vol. 31F, O-4.001.

[8] L. M. Kovrizhnykh, Plasma Phys. Rep. 25, 760 (1999).

[9] K. C. Shaing, Phys. Plasmas 10, 1443 (2003).

[10] J.-K. Park, A.H. Boozer, and J.E. Menard, Phys. Rev. Lett. 102, 065002 (2009).

[11] M. Yoshida et al., Plasma Phys. Controlled Fusion 48, 1673 (2006).

[12] P. de Vries et al., Nucl. Fusion 48, 035007 (2008).

[13] A. Salmi et al., Contrib. Plasma Phys. 48, 77 (2008).

[14] C. R. Negus et al., Rev. Sci. Instrum. 77, 10F102 (2006).

[15] Y. B. Kim, P. H. Diamond, and R. J. Groebner, Phys. Fluids B 3, 2050 (1991).

[16] C. Giroud et al., Rev. Sci. Instrum. 79, 10F525 (2008).

[17] L.-G. Eriksson, E. Righi, and K.-D. Zastrow, Plasma Phys. Controlled Fusion 39, 27 (1997).

[18] J.-M. Noterdaeme et al., Nucl. Fusion 43, 274 (2003).

[19] L.-G. Eriksson et al., Plasma Phys. Controlled Fusion 51, 044008 (2009).

[20] M.F. F. Nave et al., in Proceedings of the 34th EPS Conference on Plasma Physics, Warsaw, 2007 (ECA, 2007, Warsaw) (European Physical Society, Warsaw, 2007), Vol. 31F, P-4.158.

[21] T. Johnson et al., in Proceedings of the 10th IAEA Technical Meeting (TM) on Energetic Particles in Magnetic Confinement Systems (Kloster Seeon, Germany, 2007).

[22] J. W. Connor and R. J. Hastie, Nucl. Fusion 13, 221 (1973).

[23] K. T. Tsang and E. A. Friedman, Phys. Fluids 19, 747 (1976).

[24] P. Yushmanov, Review of Plasma Physics (Consultants Bureau, New York, 1990), Vol. 16.

[25] E. Trier et al., Nucl. Fusion 48, 092001 (2008).

[26] A. M. Garofalo et al., Phys. Rev. Lett. 101, 195005 (2008).

[27] W. Zhu et al., Phys. Rev. Lett. 96, 225002 (2006).

[28] A. J. Cole, C. C. Hegna, and J. D. Callen, Phys. Rev. Lett. 99, 065001 (2007).

[29] T. Tala et al., Phys. Rev. Lett. 102, 075001 (2009).

[30] F. Romanelli et al., in Proceedings of the 22nd Fusion Energy Conference, Geneva, Switzerland, 2008 (IAEA, Vienna, 2008). [All members of the JET-EFDA Collaboration (JET-EFDA, Culham Science Centre, Abingdon, U.K.) appear in the appendix of this paper.] 